\title{
Low-frequency variability of pulsars
}

\author{
Roger Blandford and Ramesh Narayan ${ }^{\star}$ Theoretical \\ Astrophysics, California Institute of Technology, Pasadena, CA 91125, USA
}

Accepted 1984 November 6. Received 1984 November 6; in original form 1984 July 13

\begin{abstract}
Summary. Several recent observational and theoretical investigations have suggested that long-wavelength $\left(\leqslant 10^{14} \mathrm{~cm}\right)$ density fluctuations in the interstellar medium may have a major influence on observations of pulsars and extragalactic radio sources. These long-wavelength fluctuations are responsible for refractive focusing (in contrast to the diffractive scintillation which is conventionally attributed to shorter wavelength fluctuations), and may cause the monthly to annual variations in pulsar flux densities observed at low frequency. Fluctuations in the angular sizes, pulse arrival times and pulse widths of pulsars should also be observable and should be cross-correlated with the flux density variations. The magnitude of these correlations, and their dependence upon the time lag and the observing wavelength, for different power-law spectra of density fluctuations are estimated. It appears that if the flux variability is interstellar in origin, then the spectrum must have a somewhat steeper logarithmic slope that the value $(\sim 11 / 3)$ given by the standard 'Kolmogorov' spectrum. Sensitive observations on selected pulsars will be able to confirm the importance of refractive effects in the interstellar medium and also determine the slope of the density fluctuation spectrum.
\end{abstract}

\section{Introduction}

The narrow-band intensity fluctuations of pulsars that occur on time-scales of order several minutes, and the smearing of pulse profiles at low frequencies have long been attributed to scattering caused by small-scale inhomogeneity in the electron density of the interstellar medium (e.g. Scheuer 1968). The standard explanation of these variations goes as follows. The observed angular radius $\theta$ of a point source is of the order of the root mean square angle through which a ray is scattered in traversing the distance $D$ between the pulsar and Earth. There is then a mean geometric time delay $t \sim D \theta^{2} / 2 c$ and an associated pulse broadening of the same order. This leads to a decorrelation bandwidth for the scintillations given by the uncertainty principle, $\Delta v \sim 1 / 2 \pi t$. The scattered radio waves create a diffraction pattern at Earth with a lateral coherence length, $b \sim \lambda / 2 \pi \theta$, where $\lambda$ is the wavelength of the radiation. The Earth moves through this diffraction

${ }^{\star}$ On leave from: Raman Research Institute, Bangalore 560080, India. 
pattern with speed $v$ (a combined velocity including the motions of the pulsar, Earth and the intervening interstellar medium), and so the corresponding decorrelation time-scale for the (diffractive) scintillations is $\Delta t_{\mathrm{dif}} \sim b / v$.

Observations on pulsars are roughly consistent with the scaling laws (e.g. Rickett 1977) $\theta \sim \lambda^{2.2} D^{0.6}$ milliarcsec, $\quad t \sim \lambda^{4.4} D^{2.2} \mu \mathrm{s}, \quad \Delta v \sim 100 \lambda^{-4.4} D^{-2.2} \mathrm{kHz}, \quad v \sim 100 \mathrm{~km} \mathrm{~s}^{-1}, \quad$ and $\Delta t_{\text {dif }} \sim 3 \lambda^{-1.2} D^{-0.6} \mathrm{~s}$ where $\lambda$ is in metres and $D$ is in kpc henceforth. There is, however, a considerable dispersion in these quantities; in particular, distant pulsars lying in the Galactic plane have systematically enhanced scattering (Manchester \& Taylor 1977; Cordes, Weisberg \& Boriakoff 1984).

The power spectrum of interstellar density fluctuations is often supposed to be a power law, $\Phi_{k} \propto k^{-\beta}, 2<\beta<4$ (e.g. Rickett 1977). A convenient way of understanding the scattering properties of such a medium is as follows. The typical mean square density fluctuation due to waves within a unit logarithmic range of wavevectors around $k \sim a^{-1}$ goes as $\delta n^{2}(a) \propto a^{\beta-3}$. The number of clouds of size $a$ encountered by a ray traversing the distance $D$ between the pulsar and the observer is proportional to $D / a$. (The proportionality factor could be quite small if strong interstellar turbulence is localized within isolated supernova remnants and $\mathrm{H}$ II regions with a small filling factor.) Hence the integrated rms fluctuation in the phase $\phi$ on scale $a$ goes as $\delta \phi(a) \sim\left[\delta n^{2}(a)\right]^{1 / 2} a r_{\mathrm{e}} \lambda(D / a)^{1 / 2} \propto a^{(\beta-2) / 2} \lambda D^{1 / 2}$, where $r_{\mathrm{e}}=e^{2} / m c^{2}$ is the classical electron radius. An equivalent way of stating this is in terms of the phase structure function

$\mathbf{D}(a) \equiv\left\langle[\phi(a)-\phi(0)]^{2}\right\rangle \sim \int_{a^{\prime}=0}^{a} a^{\prime \beta-2} \lambda^{2} D d\left(\ln a^{\prime}\right) \sim a^{\beta-2} \lambda^{2} D$,

which measures the mean square phase difference between two paths with a lateral separation $a$.

Consider next the rms angular scattering due to a given scale $a$, which goes as $\delta \theta(a) \sim \delta \phi(a) \lambda / a \sim a^{(\beta-4) / 2} \lambda^{2} D^{1 / 2}$. For $\beta<4$, the scattering increases at smaller scales. Nevertheless, there is an effective cut-off at very small scales since, in order for fluctuations on scale $a$ to contribute to strong scintillation, the phase difference along two paths with a separation $a$ must exceed $\sim \pi$. Thus only length scales $a \gtrsim a_{\min }$, where $\mathbf{D}\left(a_{\min }\right) \sim \pi^{2}$, i.e. $a_{\min } \sim \lambda^{-2 /(\beta-2)}$, can contribute to strong scintillation. The angular radius $\theta$ of the image can then be estimated by integrating the scattering over all scales greater than $a_{\min }$ up to the size of the image, i.e.

$\theta^{2} \sim \int_{a=a_{\min }}^{a_{\max }}[\delta \theta(a)]^{2} d(\ln a)$

or $\theta \sim \lambda^{\beta /(\beta-2)} D^{1 /(\beta-2)}$. (The upper limit can be set to $\infty$ when $\beta<4$ since the scattering is dominated by small scales; but if $\beta>4$ as we advocate later, the large scales dominate the scattering, and the upper limit should be taken to be $a_{\max } \sim D \theta$ with $a_{\min } \sim 0$.) If we use the observed scaling, $\theta \propto \lambda^{2.2}$, then $\beta$ is of the order of the Kolmogorov spectrum value of $11 / 3$ (Rickett 1977), just consistent with the assumption that $\beta<4$. Numerically, $a_{\min } \sim 10^{10} \lambda^{-1.2} D^{-0.6} \mathrm{~cm}$. Note that the transverse separation of typical rays from the image, $\sim D \theta \sim 10^{13} \lambda^{2.2} D^{1.6} \mathrm{~cm}$, exceeds $a_{\min }$ as long as $\lambda \geq \lambda^{*} \sim 0.1 D^{-0.6}$ and so there is multipath propagation - an additional requirement for strong scintillation - for wavelengths larger than $\lambda^{*}$.

The above (standard) argument indicates that the scattering $\delta \theta(a)$ contributed by length scales $\sim a$ goes as $a^{-1 / 6}$. Thus, although short length scales $a \sim a_{\min }$ contribute the majority of the scattering, the influence of the longer length scales $a_{\min } \ll a \leqslant D \theta \equiv a_{\max }$ may not be negligible. (We ignore the possibility of an outer scale smaller than $D \theta$ in the turbulence spectrum.) The influence of these larger scale density fluctuations in the interstellar medium has long been suspected; they were invoked by Shishov (1974), Hewish (1980) and others to account for the observed drifting of scintillation patterns in frequency through refractive effects. Now, refraction is dominated by fluctuations on scales $\sim a_{\max }$ which will partially focus or defocus the diffuse image of the 


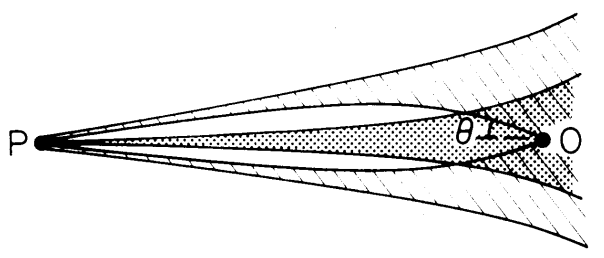

(a)

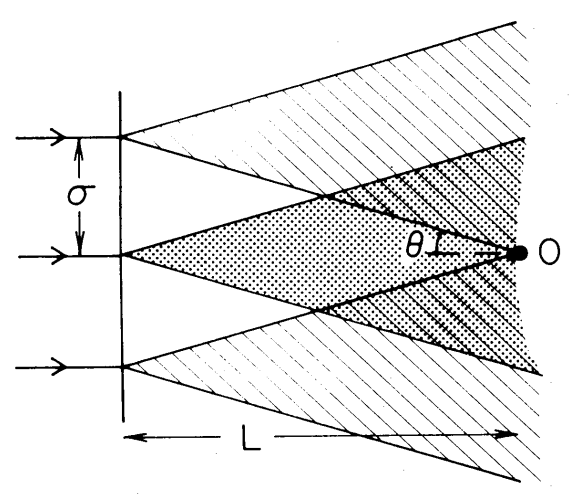

(c)

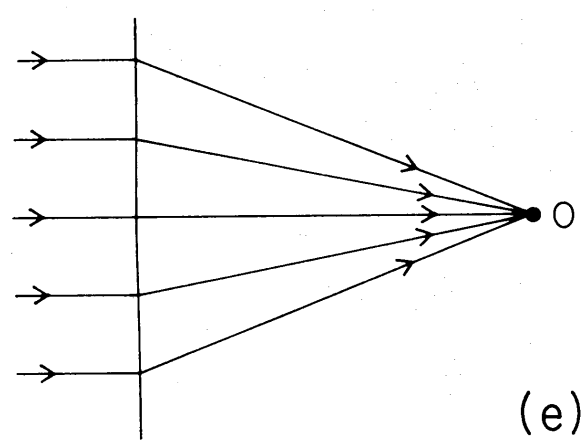

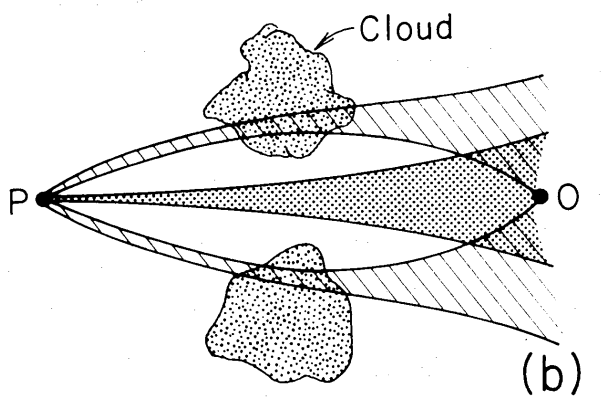
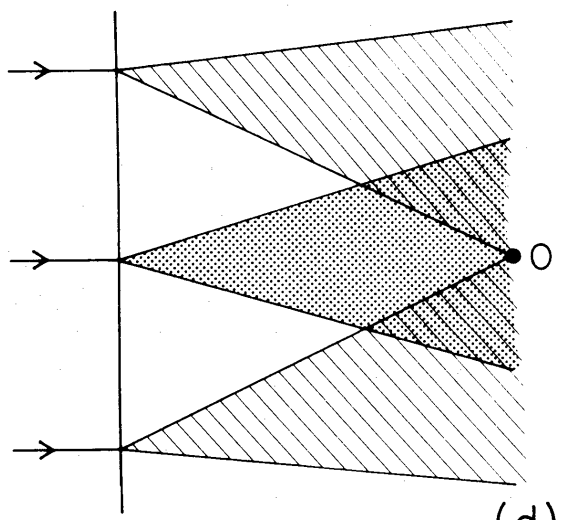

(d)

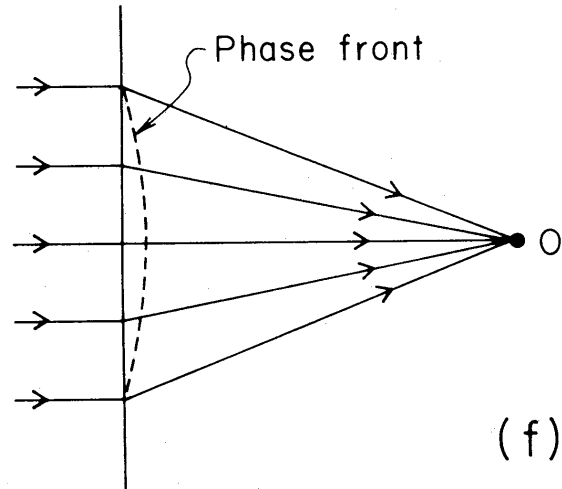

Figure 1. Schematic illustration of the effects of interstellar refraction. (a) Rays from the pulsar P are scattered by small-scale irregularities in the interstellar medium into a conical beam with angular radius $\theta$ at the observer $O$. (b) Large-scale density inhomogeneities steer the beams to focus them at $\mathrm{O}$, increasing the observed angular size and flux density. (c) Screen, equivalent to (a), in which all the scattering is localized at a distance $L$ from $O$. The average angular radius of the beam is $\sigma / L$. (d) Superposition of refraction at the screen, equivalent to (b). (e) Pulse broadening and delay due to lengthened geometrical path of scattered rays. (f) Dispersive contribution to the pulse broadening and delay. If there is a density excess centred on the beam, the phase fronts will be advanced at the centre of the beam and the pulses, which propagate at the group velocity, will be retarded.

pulsar (see Fig. 1). This will cause the observed flux to change on time-scales $\Delta t_{\text {ref }} \sim a_{\text {max }} / v \sim 10^{6} \lambda^{2.2} D^{1.6} v_{7}^{-1} \mathrm{~s}$ where $v=100 v_{7} \mathrm{~km} \mathrm{~s}^{-1}$. In fact, long-term variation of just this type is common among pulsars (Cole, Hesse \& Page 1970; Helfand, Fowler \& Kuhlman 1977; Sieber 1982). In particular, all three components of the Crab pulsar (main pulse, interpulse and precursor) are known to vary in phase at a wavelength of $1 \mathrm{~m}$ (Rankin, Payne \& Campbell 1974).

This explanation for the long-term variability of pulsars has been given explicitly by Rickett, Coles \& Bourgois (1984) and partly by earlier authors referred to therein. The explanation is compelling because it successfully explains the scaling of the variability time-scale with pulsar distance and the wavelength of observation. Furthermore, as these authors speculate, it may also account for the phenomenon of low-frequency variability in extragalactic radio sources. 
In this paper, we present a model (a simplified version of one introduced by Shishov 1974) which allows us to relate the observed intensity variation of a point radio source like a pulsar to the strength of the interstellar fluctuation spectrum when the focusing effects are not too large. We then use this model to show that there should also be variability in the arrival times and widths of pulses from radio pulsars, as well as in the observed angular sizes. Furthermore, there should be correlations among the fluctuations of all these parameters. We give general expressions for the various auto- and cross-correlations and present results for the specific choice of a power-law spectrum for the density fluctuations. Finally we estimate the order of magnitude of the expected fluctuations in the various observables and discuss the feasibility of looking for the effects we describe in pulsars and compact extragalactic radio sources. On the basis of our estimates, we conclude that if refractive scintillation is to account for the long-term flux variability of pulsars and the low-frequency variable extragalactic radio sources, then the power spectrum of density fluctuations should have a slope $\beta \geq 4$, somewhat in excess of the Kolmogorov value. Roberts \& Ables (1982) reached a similar conclusion on the basis of their observations on dynamic pulsar scintillation spectra.

After this work was largely completed, we obtained a copy of an unpublished thesis written by R. V. E. Lovelace in 1970. Several of the qualitative ideas contained in the present paper are also explored in this thesis. However, the calculation technique presented here is believed to be original and the observational data base has increased dramatically since 1970 . This allows us to give quantitative estimates of the influence of refractive scintillation.

A more detailed discussion of intensity fluctuations, including the case $\beta>4$, is contained in Goodman \& Narayan (1985).

\section{The scattering model}

We idealize both the short-wavelength ('diffractive') and the long-wavelength ('refractive') fluctuations by regarding them as distinct entities, rather than as two regions of an extensive turbulence spectrum. This approximation should be valid so long as the length scales associated with the two phenomena, $a_{\min }$ and $a_{\max }$, are well separated. Fig. 1 shows qualitatively the effects we are studying. A bundle of rays leaving the pulsar in a given direction spreads out because of the scattering by small-scale inhomogeneities and reaches the observer plane over an extended area. Consequently, at a given point on the observer plane, rays are received from a range of directions (Fig. 1a). If we neglect large-scale fluctuations for the moment and average over a time much greater than the scintillation time-scale $\Delta t_{\text {dif }}$, the image of the pulsar will be essentially circular with a characteristic angular radius $\theta$. We approximate the intensity distribution over this 'seeing disc' by a Gaussian with angular radius $\theta$, although in actual fact there is a power-law tail, $I\left(\theta^{\prime}\right) \propto \theta^{\prime-\beta}, \theta^{\prime} \gg \theta$. The error from this approximation is expected to be very small since the Gaussian gives a good fit down to 1 per cent of the intensity maximum (see fig. 1 of Woolf 1982).

Next, for simplicity we replace the distributed density fluctuations between the source and the observer by a single scattering screen located a distance $L$ from Earth (Fig. 1c). In this 'equivalent' geometry, we regard the source as being very distant compared with $L$ so that the incident radiation from the source can be modelled as plane waves. We believe that the inaccuracy introduced by this approximation is no greater than that already inherent in the problem through our ignorance of the distribution of scattering along the line-of-sight to the pulsar. Given a model of the scattering medium, we fix the distance $L$ of the equivalent screen and adjust the strength of its scattering so that the observed angular size $\theta$ of a point source as well as the mean geometrical time delay $\left(t=L \theta^{2} / 2 c\right)$ are the same as with the model of the medium. Appendix A discusses how we do this. We define $\sigma=L \theta$ to be the linear size of the image when projected on the equivalent screen. 
Fig. 1(b) and (d) show the effect of the large-scale refractive inhomogeneity in the scattering, which again we take to be localized to the equivalent screen. The effect is primarily to steer the local Gaussian angular intensity distribution to a new direction determined by the local phase gradient of the refractive fluctuation. Depending on whether there is focusing or defocusing, one has either an increase or decrease in the received flux and the image size. There are similar fluctuations in the time delay and pulse width caused by variations in the image size (Fig. 1e) as well as the propagation velocity of the radiation (Fig. 1f).

\section{Expressions for the fluctuations}

We treat the refractive inhomogeneity in the weak deflection limit, i.e., we assume that the rms deflection due to this is smaller than the rms diffractive scattering angle $\sigma / L$. It is convenient to work with the phase change along an unscattered ray induced by the density fluctuations $\delta n$ in the refractive inhomogeneity

$\phi=r_{\mathrm{e}} \lambda \int_{0}^{D} d z \delta n(z)$

In line with our thin-screen approximation we localize this $\phi$ to the screen. The associated extra bending angle $\boldsymbol{\eta}(\mathbf{r})$ of a ray at transverse location $\mathbf{r}$ on the screen is then given by

$\boldsymbol{\eta}(\mathbf{r})=-\chi[\partial \phi(\mathbf{r}) / \partial \mathbf{r}]$

where $x=\lambda / 2 \pi$.

We now compute the observed flux near Earth by integrating the received itensity over the solid angle subtended by the equivalent screen. In order to compute the time dependence due to the relative motion between the observer, the screen and the Earth, we calculate the flux at a general point $\mathbf{x}$ in the observer plane perpendicular to the line-of-sight (see Fig. 2). If $\bar{F}$ is the mean flux from the source, then the intensity received at $\mathbf{x}$ from unit area around the point $\mathbf{x}+\mathbf{r}$ on the screen is

$I(\mathbf{r}, \mathbf{x})=\frac{\bar{F}}{\pi \sigma^{2}} \exp -\left[\frac{L \boldsymbol{\eta}+\mathbf{r}}{\sigma}\right]^{2}$

where $\boldsymbol{\eta}$ is evaluated at the point $(\mathbf{x}+\mathbf{r})$. As the deflection $L \boldsymbol{\eta}$ is, by assumption, small compared with $\sigma$, we expand the argument of the exponential to first order,

$I(\mathbf{r}, \mathbf{x})=\frac{\bar{F}}{\pi \sigma^{2}} \exp \left(-\frac{r^{2}}{\sigma^{2}}\right)\left[1-\frac{2 L \boldsymbol{\eta} \cdot \mathbf{r}}{\sigma^{2}}\right]$.

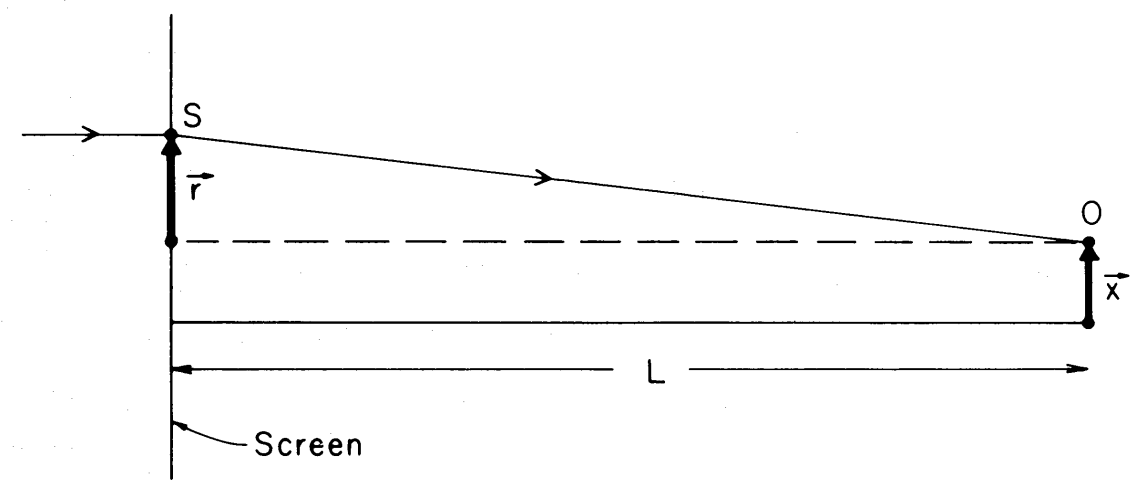

Figure 2. Scattering geometry with the equivalent screen. Rays from the pulsar are scattered at the point $\mathrm{S}$ on the screen located at a displacement $\mathbf{x}+\mathbf{r}$ from an arbitrary origin, to be received by the observer $\mathrm{O}$ at a displacement $\mathbf{x}$. 
The fluctuation in the observed radio flux is then given to first order by

$\frac{\Delta F(\mathbf{x})}{\bar{F}}=-\frac{2 L}{\pi \sigma^{4}} \int d^{2} r(\boldsymbol{\eta} \cdot \mathbf{r}) \exp \left(-\frac{r^{2}}{\sigma^{2}}\right)$

Substituting for $\boldsymbol{\eta}$ from equation (3.2) and integrating by parts we obtain the normalized intensity fluctuation to be

$\delta F(\mathbf{x}) \equiv \frac{\Delta F}{\bar{F}}=\frac{4 \lambda L}{\pi \sigma^{6}} \int d^{2} r \phi\left(r^{2}-\sigma^{2}\right) \exp \left(-\frac{r^{2}}{\sigma^{2}}\right)$

with $\phi=\phi(\mathbf{x}+\mathbf{r})$.

We define the mean angular area of the image to be

$\Omega(\mathbf{x})=\frac{\pi \int d^{2} r r^{2} I(\mathbf{r}, \mathbf{x})}{L^{2} \int d^{2} r I(\mathbf{r}, \mathbf{x})}$.

The mean value of $\Omega$ is $\pi \sigma^{2} / L^{2}$ and the normalized fluctuation about the mean is

$\delta \Omega(\mathbf{x}) \equiv \frac{L^{2} \Omega}{\pi \sigma^{2}}-1=-\frac{2 L}{\pi \sigma^{6}} \int d^{2} r\left(r^{2}-\sigma^{2}\right)(\boldsymbol{\eta} \cdot \mathbf{r}) \exp \left(-\frac{r^{2}}{\sigma^{2}}\right)$

where we have retained only terms linear in the phase fluctuation. Simplifying as before we obtain

$\delta \Omega(\mathbf{x})=\frac{4 \chi L}{\pi \sigma^{8}} \int d^{2} r \phi\left(r^{4}-3 r^{2} \sigma^{2}+\sigma^{4}\right) \exp \left(-\frac{r^{2}}{\sigma^{2}}\right)$.

A further quantity that can be measured is the mean time delay associated with the refractive density fluctuations. There are two contributions to this: a geometric time delay $t_{\text {geom }}$ from extra path length and a dispersive time delay $t_{\text {disp }}$ due to reduced group velocity. Combining both contributions, the signal from the point $\mathbf{x}+\mathbf{r}$ is delayed with respect to the mean arrival of the central ray from $\mathbf{x}$ by an amount

$t(\mathbf{r}, \mathbf{x})=\frac{r^{2}}{2 L c}+\frac{\rtimes \phi}{c}$

where we have allowed for the fact that a radio pulse with advanced phase (and hence an increased phase velocity) has a reduced group velocity. The mean time delay of the pulse, i.e. the time delay corresponding to the centroid of the pulse profile, is

$t_{0}=\sigma^{2} / 2 L c$.

and the normalized fluctuation in the delay is

$\delta t(\mathbf{x}) \equiv \frac{\Delta t(\mathbf{x})}{t_{0}}=\delta \Omega(\mathbf{x})+\frac{2 L \chi}{\pi \sigma^{4}} \int d^{2} r \phi \exp \left(-\frac{r^{2}}{\sigma^{2}}\right)$.

A related quantity is the pulse width $\tau$, which will also fluctuate. We define $\tau(\mathbf{x})$ as follows

$\tau^{2}(\mathbf{x}) \equiv\left[t^{2}(\mathbf{x})\right]-[t(\mathbf{x})]^{2}=\frac{\int d^{2} r t^{2}(\mathbf{r}, \mathbf{x}) I(\mathbf{r}, \mathbf{x})}{\int d^{2} r I(\mathbf{r}, \mathbf{x})}-\left[\frac{\int d^{2} r t(\mathrm{r}, \mathbf{x}) I(\mathbf{r}, \mathbf{x})}{\int d^{2} r I(\mathbf{r}, \mathbf{x})}\right]^{2}$.

The mean pulse width is then

$\tau_{0}=\sigma^{2} / 2 L c=t_{0}$ 
and the normalized fluctuation in the width is

$\delta \tau(\mathbf{x}) \equiv \frac{\Delta \tau}{\tau_{0}}=\frac{2 \chi L}{\pi \sigma^{10}} \int d^{2} r \phi\left(r^{6}-5 r^{4} \sigma^{2}+5 r^{2} \sigma^{4}-\sigma^{6}\right) \exp \left(-\frac{r^{2}}{\sigma^{2}}\right)$

where only terms linear in $\phi$ have been retained in $t^{2}(\mathbf{x})$ and $[t(\mathbf{x})]^{2}$ and we use $\Delta \tau \sim \Delta\left(\tau^{2}\right) / 2 \tau_{0}$.

\section{Correlations and power spectrum}

In the previous section we obtained expressions for the normalized fluctuations about their mean values of various observable quantities, $F, \Omega, t$ and $\tau$, using the equivalent screen model. Because of the motion of the source, observer and the medium, the screen will move with respect to the Earth-source line, causing these parameters to vary. We now estimate the correlations of these quantities with themselves and each other.

As we are continually sampling fresh interstellar medium, an average over time, denoted by $\langle\ldots\rangle$, is equivalent to an average over space and will be treated as such. We assume that the random motion of the interstellar density inhomogeneities is much slower than the transverse speed of the Solar System through the diffraction pattern, which we denote by $\mathbf{v}$. Two observations made a time $T$ apart will then correspond to sampling two areas on the screen a distance $\mathbf{s}=\mathbf{v} T$ apart. Let $A_{1}$ and $A_{2}$ be any two of the four fluctuations, $\delta F . \delta \Omega, \delta t, \delta \tau$. The fluctuations $\delta A_{1}\left(\mathbf{x}, x_{1}\right)$ and $\delta A_{2}\left(\mathbf{x}+\mathbf{s}, x_{2}\right)$ at two points separated by $\mathbf{s}$ and measured at wavelengths $x_{1}$ and $x_{2}$ are of the general form [see equations (3.6), (3.8), (3.12) and (3.15)]

$\delta A_{1}\left(\mathbf{x}, \chi_{1}\right)=\int d^{2} r \phi\left(\mathbf{x}+\mathbf{r}, \chi_{1}\right) f_{1}\left(r, \chi_{1}\right)$

$\delta A_{2}\left(\mathbf{x}+\mathbf{s}, \chi_{2}\right)=\int d^{2} r \phi\left(\mathbf{x}+\mathbf{s}+\mathbf{r}, \chi_{2}\right) f_{2}\left(r, \chi_{2}\right)$

where we note that $f_{1}$ and $f_{2}$ depend on the respective $\chi$ both explicitly and implicitly through $\sigma$. The product of the two fluctuations is

$\delta A_{1}\left(\mathbf{x}, \chi_{1}\right) \delta A_{2}\left(\mathbf{x}+\mathbf{s}, \chi_{2}\right)=\int d^{2} r_{1} d^{2} r_{2} \phi\left(\mathbf{x}+\mathbf{r}_{1}, x_{1}\right) \phi\left(\mathbf{x}+\mathbf{s}+\mathbf{r}_{2}, \chi_{2}\right) f_{1}\left(r_{1}, \chi_{1}\right) f_{2}\left(r_{2}, x_{2}\right)$.

Take two-dimensional Fourier transforms and average over all $\mathbf{x}$ keeping $\mathbf{s}$ constant to obtain the mean correlation

$\left\langle\delta A_{1}\left(\mathbf{x}, \chi_{1}\right) \delta A_{2}\left(\mathbf{x}+\mathbf{s}, \chi_{2}\right)\right\rangle=\chi_{1} \chi_{2} \int \frac{d^{2} q}{(2 \pi)^{2}} \tilde{f}_{1}\left(\mathbf{q}, \chi_{1}\right) \tilde{f}_{2}^{*}\left(\mathbf{q}, \chi_{2}\right) Q(\mathbf{q}) \exp (i \mathbf{q} \cdot \mathbf{s})$,

where

$\tilde{f}_{i}(\mathbf{q}, \lambda)=\int d r^{2} f_{i}(r, \lambda) \exp (-i \mathbf{q} \cdot \mathbf{r})$

$Q(\mathbf{q})=\left\langle\frac{1}{x_{1}} \tilde{\phi}\left(\mathbf{q}, x_{1}\right) \frac{1}{x_{2}} \tilde{\phi}^{*}\left(\mathbf{q}, x_{2}\right)\right\rangle / A$

$\tilde{\phi}(\mathbf{q}, \chi)=\int d^{2} r \phi(\mathbf{x}+\mathbf{r}, \chi) \exp (-i \mathbf{q} \cdot \mathbf{r})$

and we use the fact that the fluctuations are real quantities. In averaging over $\mathbf{x}$ we have made the standard approximation that the phases of different $\bar{\phi}(\mathbf{q})$ are uncorrelated with each other and that the total screen area is $A$, chosen so that $\sigma^{2} \ll A$. If we further assume that the power spectrum 
$Q(\mathbf{q})$ is isotropic we can simplify equation (4.4) to the one-dimensional integral

$\left\langle\delta A_{1}\left(\mathbf{x}, \chi_{1}\right) \delta A_{2}\left(\mathbf{x}+\mathbf{s}, \chi_{2}\right)\right\rangle=\chi_{1} \chi_{2} \int \frac{d q}{2 \pi} \tilde{f}_{1}\left(q, \chi_{1}\right) \tilde{f}_{2}\left(q, \chi_{2}\right) q Q(q) J_{0}(q s)$.

Given the power spectrum $Q(q)$ of the refractive fluctuations, equation (4.8) can be used to prédict the correlations between any two of the pulsar parameters that we have considered, by means of the following expressions for the respective Fourier transforms

$\tilde{f}_{F}(q, \lambda)=-\frac{\lambda \mathrm{L}}{\sigma^{2}} q^{2} \sigma^{2} \exp \left(-\frac{1}{4} q^{2} \sigma^{2}\right)$

$\tilde{f}_{\Omega}(q, \lambda)=-\frac{\chi L}{\sigma^{2}}\left(q^{2} \sigma^{2}-\frac{1}{4} q^{4} \sigma^{4}\right) \exp \left(-\frac{1}{4} q^{2} \sigma^{2}\right)$

$\tilde{f}_{t}(q, \lambda)=\frac{2 \chi L}{\sigma^{2}}\left(1-\frac{1}{2} q^{2} \sigma^{2}+\frac{1}{8} q^{4} \sigma^{4}\right) \exp \left(-\frac{1}{4} q^{2} \sigma^{2}\right)$

$\tilde{f}_{r}(q, \lambda)=-\frac{3 \rtimes L}{2 \sigma^{2}}\left(q^{2} \sigma^{2}-\frac{1}{3} q^{4} \sigma^{4}+\frac{1}{48} q^{6} \sigma^{6}\right) \exp \left(-\frac{1}{4} q^{2} \sigma^{2}\right)$.

In fact, given measurements of the fluctuation correlation functions we can invert equation (4.8) to compute the associated power spectrum of the phase fluctuations

$\left\langle\tilde{\phi}\left(\mathbf{q}, x_{1}\right) \tilde{\phi}^{*}\left(\mathbf{q}, \chi_{2}\right)\right\rangle=\frac{2 \pi}{\tilde{f}_{1}\left(\mathbf{q}, \chi_{1}\right) \tilde{f}_{2}\left(\mathbf{q}, \chi_{2}\right)} \int d s\left\langle\delta A_{1}\left(\mathbf{x}, \chi_{1}\right) \delta A_{2}\left(\mathbf{x}+\mathbf{s}, \chi_{2}\right)\right\rangle s J_{0}(q s)$.

It is not clear how useful these inversions will be in practice.

Now consider the case $\lambda_{1}=\lambda_{2}$ and assume that the spectrum of density fluctuations is a power law

$Q(q)=Q_{0} q^{-\beta}$.

Note that $Q_{0}$ is related to $C_{\mathrm{N}}^{2}$ of Rickett (1977) by

$Q_{0}=\alpha(2 \pi)^{5} r_{\mathrm{e}}^{2} \int_{0}^{D} d z C_{\mathrm{N}}^{2}$

where the numerical factor $\alpha$ (of order unity) is necessary to correct for small differences in the assumptions regarding the equivalent screen. We consider two values for the exponent $\beta$ in this paper, the 'Kolmogorov' value $\beta=11 / 3$ and the 'critical' value $\beta=4$. In the former case, the angular size of the source attributable to density inhomogeneity on the diffractive scale exceeds the effective scattering due to the refractive scale by a factor $\sim\left(q_{\max } / q_{\min }\right)^{1 / 6} \sim\left(\sigma / a_{\min }\right)^{1 / 6}$. In the latter case, the factor is $\sim \ln \left(q_{\max } / q_{\min }\right)$. The Taylor expansion in equation (3.4) is acceptably accurate for our present approximate calculations as long as $q_{\max } \gtrsim 100 q_{\min }$.

We substitute equation (4.14) into equations (4.8) and (4.9) and use standard results (e.g. Abramowitz \& Stegun 1970) to obtain the autocorrelation of the intensity fluctuations

$\langle\delta F(\mathbf{x}) \delta F(\mathbf{x}+\mathbf{s})\rangle=K h_{1}(s)$ 
where

$K=\frac{Q_{0} \star^{4} L^{2}}{2 \pi \sigma^{(6-\beta)}}$

$\left.h_{n}(s)=2^{n+1-\beta / 2} \Gamma\left(n+2-\frac{\beta}{2}\right) M\left[n+2-\frac{\beta}{2}, 1,-\frac{s^{2}}{2 \sigma^{2}}\right)\right]$

and $M(a, b, x)$ is the confluent hypergeometric function. The constant $K$ measures the strength of the fluctuations. It decreases with increasing wavelength as the focusing power of the scale $\sigma$ decreases.

Similarly, the cross-correlation between the intensity and image size fluctuations is given by

$\langle\delta F(\mathbf{x}) \delta \Omega(\mathbf{x}+\mathbf{s})\rangle=K\left[h_{1}(s)-1 / 4 h_{2}(s)\right]$.

Expressions are given for the 10 correlations among the four observable parameters we have considered in Appendix B. Numerical evaluations of these expressions are contained in Figs 3 and 4.

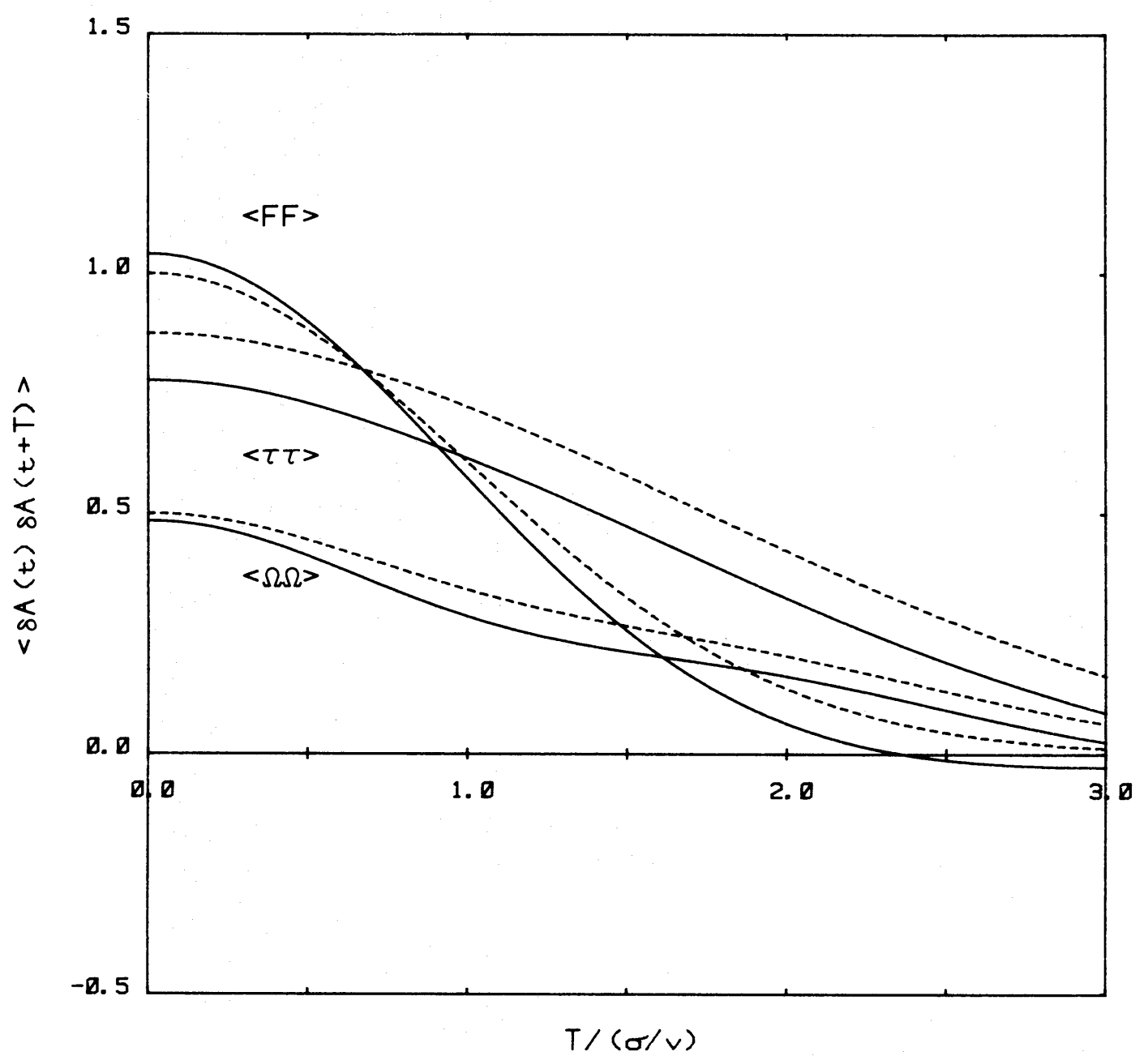

Figure 3. Autocorrelation functions for relative fluctuations in flux density, angular area and pulse width for 'Kolmogorov' ( $\beta=11 / 3$, bold lines) and 'critical' $(\beta=4$, dashed lines) spectra of density fluctuations. The autocorrelation functions are measured in terms of the dimensionless constant $K$ defined in equations (4.17), (4.25) and as functions of time difference, measured in units of $\sigma / v$. The expressions evaluated are given in Appendix B. 


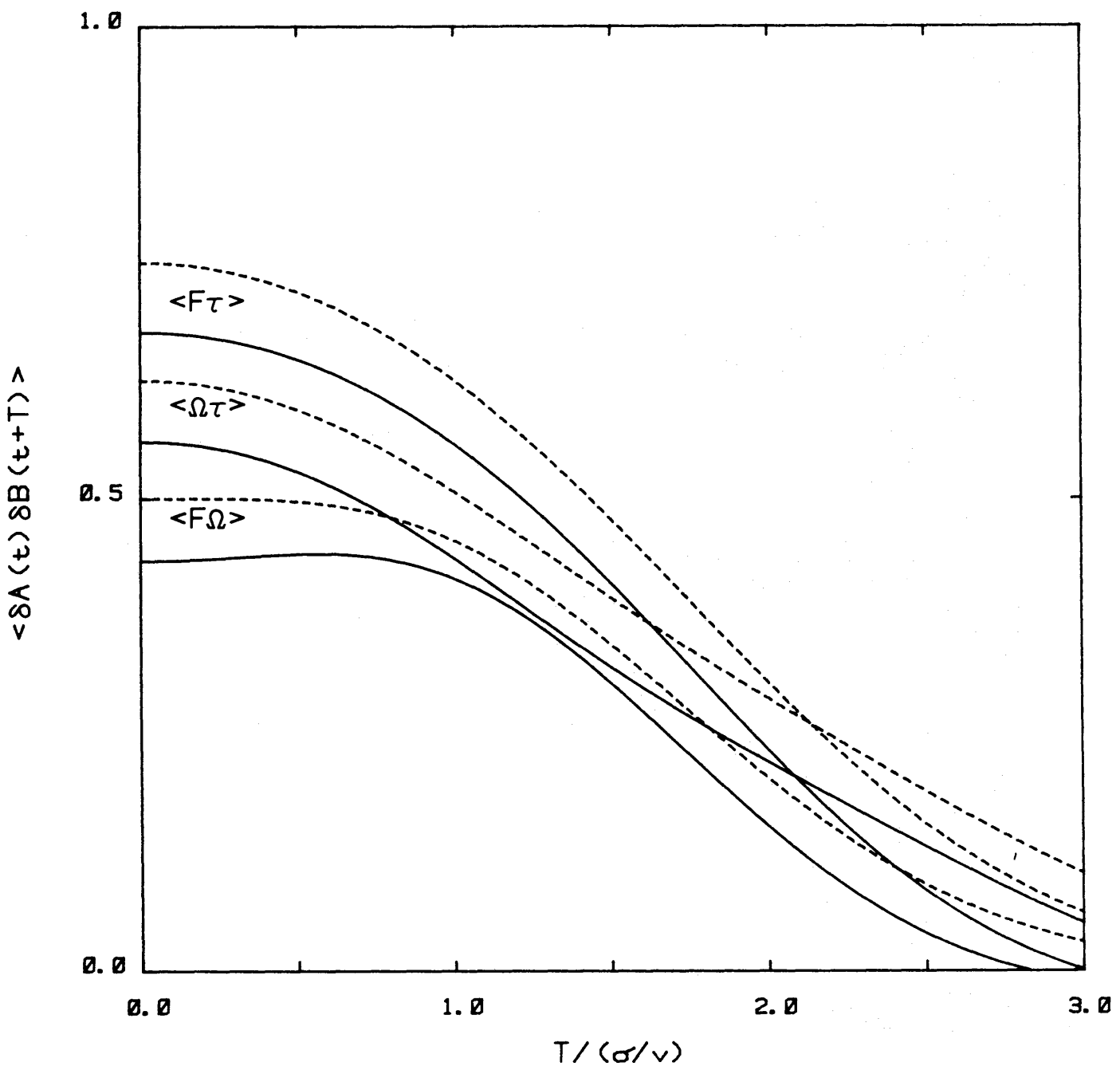

Figure 4. Cross-correlation functions for relative fluctuations in flux density $(F)$, angular area $(\Omega)$ and pulse width $(\tau)$ for 'Kolmogorov' and 'critical' spectra as in Fig. 3. Finite cross-correlation coefficients with the arrival time fluctuations can be computed when there is a Kolmogorov density fluctuation spectrum (Appendix B). These are not useful, because the arrival-time autocorrelation function diverges, and are therefore not plotted.

We can also explore the correlations in wavelength. We substitute expressions (4.9), (4.10), and (4.12) in equation (4.8) with $s=0$ to obtain

$\left\langle\delta F\left(\lambda_{1}\right) \delta F\left(\lambda_{2}\right)\right\rangle \propto \frac{\lambda_{1}^{2} \lambda_{2}^{2}}{\left(\sigma_{1}^{2}+\sigma_{2}^{2}\right)^{(6-\beta) / 2}}$.

Expressions for $\left\langle\delta \Omega\left(\lambda_{1}\right) \delta \Omega\left(\lambda_{2}\right)\right\rangle$ and $\left\langle\delta \tau\left(\lambda_{1}\right) \delta \tau\left(\lambda_{2}\right)\right\rangle$ are also given in Appendix B and displayed in Fig. 5. We confirm that broad-band variations should be seen.

We can now relate $K$ to the angular scattering measured by $\sigma$, assuming that the power spectrum, equation (4.14), extends down to short length scales. These small-scale fluctuations will only be effective at scattering a ray so long as they contribute a total phase change $\Delta \phi \geq \pi$ (see for example Gapper \& Hewish 1981). We can then define a shortest scale for scattering by truncating the spectrum at $q=q_{\max }$. For $\beta=11 / 3$, we have

$\left\langle\phi^{2}\right\rangle=\chi^{2} \int_{q_{\max }}^{\infty} \frac{d^{2} q}{(2 \pi)^{2}} Q(q)=\pi^{2}$ 


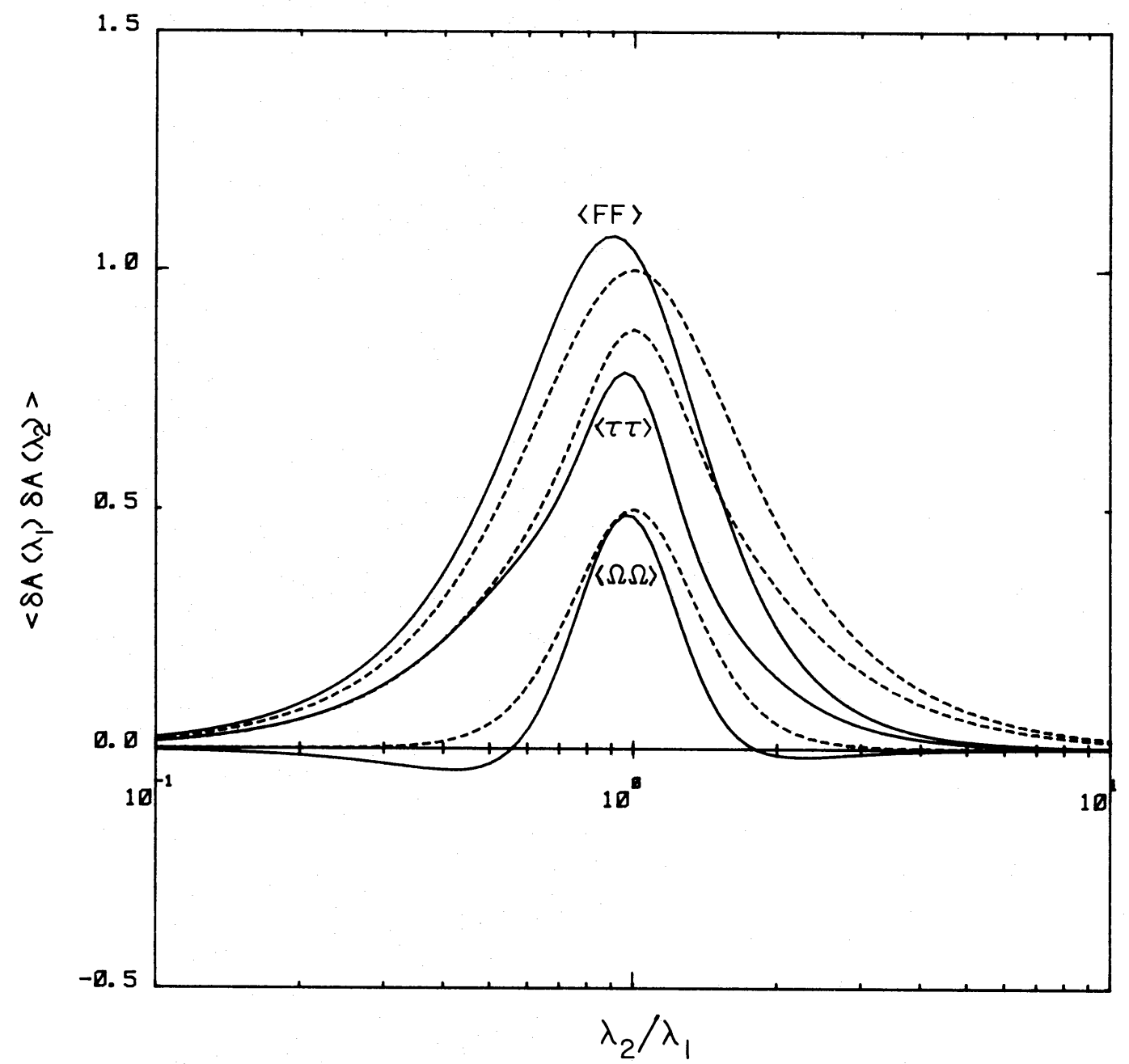

Figure 5. Autocorrelation functions for simultaneous fluctuations in the flux density $F$, angular area $\Omega$ and pulse width $\tau$ at two different wavelengths $\lambda_{1}, \lambda_{2}$ and variation with wavelength ratio $\lambda_{2} / \lambda_{1}$. The bold lines correspond to a 'Kolmogorov' spectrum and the dashed lines to a 'critical' spectrum.

$q_{\max }=\left[\frac{3 \star^{2} Q_{0}}{10 \pi^{3}}\right]^{3 / 5}$.

The mean angular area of the image is now given by

$\langle\Omega\rangle=\pi\left\langle\theta^{2}\right\rangle=\frac{\pi \sigma^{2}}{L^{2}}=\pi \star^{4} \int_{\sigma^{-1}}^{q_{\max }} \frac{d^{2} q}{(2 \pi)^{2}} q^{2} Q(q)$,

where the integral is cut off below $q=\sigma^{-1}$ because the corresponding spatial wavelengths are larger than $\sigma$ and do not contribute to the image size. (They do, however, contribute to image wander.) If $q_{\max } \gg q_{\min }$, then for $\beta<4, q_{\min }$ can be taken to be 0 . Hence, substituting for $\sigma^{2}$ in equation (4.17), we find

$K=1.1 \star^{-17 / 15} Q_{0}^{-2 / 5} L^{-1 / 3}$.

For $\beta=4$, we retain the lower limit in (4.23). We find that $K$ solves

$K^{-1}=\ln \left(\frac{Q_{0} \star^{3} L}{2^{3 / 2} \pi^{2} K^{1 / 2}}\right)$. 
We should also consider the possibility that the density fluctuation spectrum may have a slope in excess of the critical value $\beta=4$. In this case, the angular scattering will be dominated by fluctuations of size $\gtrsim \sigma$, and the assumption that the focusing effects are small, which underlies our calculation of the expected variability, is no longer strictly valid. The integral in equation (4.23) for the scattering angle will now be dominated by the lower cut-off at $q_{\min } \sim \sigma^{-1}$. This cut-off is crucial if one is to obtain a finite answer. Waves with $q<q_{\min }$ have infinite scattering power as $q \rightarrow 0$, but they only contribute to image wander, which formally diverges, and do not affect the size of the image. Replacing $q_{\max }$ by $\infty$, we obtain

$\sigma=\left[\frac{Q_{0}}{2 \pi(\beta-4)}\right]^{1 /(6-\beta)} \lambda^{4 /(6-\beta)} L^{2 /(6-\beta)}$.

Note that the observed scaling of the angular size with wavelength, namely $\theta=\sigma / L \propto \lambda^{2.2}$ can be just as well reproduced by a spectrum of slope $\beta=4.2$ as by a Kolmogorov spectrum. Although we cannot compute the correlation coefficients in the case $\beta>4$, we can see that they will be non-linear [i.e. of magnitude $\mathrm{O}(1)$ ] and that they should have the same sense and roughly the same dependence upon $s$ as the expressions computed for $\beta \leqslant 4$.

\section{Applications to pulsars}

In the preceding sections we have described a simple, approximate model of interstellar scattering that separates the small-scale density variations responsible for diffraction from the large-scale variations responsible for the refractive effects and used it to show that fluctuations in the intensity, the angular size, the arrival time and the pulse width should be correlated. In this section we use present knowledge of the interstellar medium to demonstrate that some of these correlations ought to be measurable in radio pulsars using existing techniques. If these correlations can be detected, then it might be possible to invert the procedure and use the observations to refine our understanding of the interstellar medium.

In the following we specialize to a uniform distribution of inhomogeneities between the pulsar and the observer, which is case (a) in Appendix A. (For case (b), which corresponds to a source well of out of the Galactic plane, one should replace $C_{-4} D$ by $3 C_{-4} H \operatorname{cosec}(b)$ and $D$ by $2 H \operatorname{cosec}(b)$ in the expressions below, where $b$ us the Galactic latitude of the source and $H$ is the scale height of the inhomogeneities in $\mathrm{kpc}$.)

\section{1 'KOLMOGOROV' SPECTRUM $(\beta=11 / 3)$}

We first assume that the power spectrum of refractive fluctuations has a Kolmogorov power-law form given by equation (4.14) with

$Q_{0}=7 \times 10^{-18} C_{-4} D \mathrm{~cm}^{-11 / 3}$

which is equivalent to $C_{\mathrm{N}}^{2}=10^{-4} C_{-4} \mathrm{~m}^{-20 / 3}$ in the notation of Armstrong, Cordes \& Ricket1 (1981). $D$ is the distance to the pulsar measured in kpc. We note that the values of $C_{-4}$ inferred from decorrelation bandwidth and pulse broadening measurements are $\sim 1$ for nearby pulsars bu1 can exceed this by factors up to $10^{4}$ for distant pulsars in the plane of the galaxy (Cordes, Weisberg $\&$ Boriakoff 1984). We substitute equation (5.1) into equation (4.23) to obtain

$\sigma=1.4 \times 10^{13} C_{-4}^{3 / 5} \lambda^{11 / 5} D^{8 / 5} \mathrm{~cm}$

where we measure the wavelength $\lambda$ in metres. We can use this value of $\sigma$ to estimate the mear source angular diameter $2 \theta$, the scattering time delay $t_{0}$, the pulse broadening $\tau_{0}$ and the 
decorrelation bandwidth $\Delta v$,

$2 \theta=4 C_{-4}^{3 / 5} \lambda^{11 / 5} D^{3 / 5}$ milliarcsec

$\tau_{0}=t_{0}=2.4 C_{-4}^{6 / 5} \lambda^{22 / 5} D^{11 / 5} \mu \mathrm{s}$

$\Delta v=70 C_{-4}^{-6 / 5} \lambda^{-22 / 5} D^{-11 / 5} \mathrm{kHz}$.

(The pulse broadening and decorrelation bandwidth are conventionally used to estimate $C_{-4}$.)

It is convenient to express the normalizing constant $K$ in equation (4.17) as

$K=\left(\lambda / \lambda^{*}\right)^{-17 / 15}$

where

$\lambda^{*}=0.05 C_{-4}^{-6 / 17} D^{-11 / 17} \mathrm{~m}$.

$\lambda^{*}$ is roughly the wavelength at which the pulsar rays are scattered into the first Fresnel zone. Our model can only be self-consistent if the angle through which a ray is refracted is somewhat smaller than the angle through which it is scattered. This in turn requires that $\lambda \geqslant 4 \lambda *$.

Substituting numerical values the rms intensity fluctuation is given by

$\delta F \equiv \frac{\Delta F}{F}=0.18 C_{-4}^{-1 / 5} \lambda^{-17 / 30} D^{-11 / 30}, \quad \lambda \gtrsim 0.2 C_{-4}^{-6 / 17} D^{-11 / 17} \mathrm{~m}$.

If we assume that the Earth moves relative to the source screen frame with a speed of $100 v_{7} \mathrm{~km} \mathrm{~s}^{-1}$, then we can use Fig. 3 to estimate the decorrelation time-scale, that is the half-power width of the autocorrelation function, for the intensity fluctuations,

$T_{\mathrm{F}}=18 C_{-4}^{3 / 5} \lambda^{11 / 5} D^{8 / 5} v_{7}^{-1}$ day.

Equation (5.9) for the fluctuation time-scale agrees within 10 per cent with that given by Rickett et al. (1984), which in turn is consistent with the data tabulated by Sieber (1982). We also find that the amplitude of the fluctuations should decrease with increasing wavelength, as is evident in the expression of Rickett et al. (1984). However, our model allows us to derive further relations. In particular, we predict that the angular size, arrival time residual and pulse-width residual should also fluctuate. The mean angular size fluctuation can be estimated through $\Delta \theta / \theta \sim \delta \Omega / 2$. Hence, from Fig. 3 and equations (4.24) and (5.3), we have

$2 \Delta \theta=0.24 C_{-4}^{2 / 5} \lambda^{49 / 30} D^{7 / 30}$ milliarcsec.

The pulse-width fluctuation can likewise be calculated to be

$\Delta \tau=0.4 C_{-4} \lambda^{23 / 6} D^{11 / 6} \mu \mathrm{s}$.

The corresponding decorrelation times are, from the half-widths in Fig. 3,

$T_{\theta}=21 C_{-4}^{3 / 5} \lambda^{11 / 5} D^{8 / 5} v_{7}^{-1}$ day

$T_{\tau}=30 C_{-4}^{3 / 5} \lambda^{11 / 5} D^{8 / 5} v_{7}^{-1}$ day.

In Appendix B we show that the arrival-time residual formally diverges. Physically this means that there is a limit to the timekeeping quality of pulsars. As pulsars are observed for longer periods of time, larger clouds can interpose themselves along the line-of-sight giving progressively larger dispersion measure fluctuations. To estimate the timing residuals we truncate the relevant integral over $Q(q)$ at $q_{\min } \sim 2 \pi / v t_{\mathrm{obs}}$, where $t_{\mathrm{obs}}$ is the length of the observing period, assumed to exceed the time $\sigma / v$ to cross the scattering disc. (For $t_{\text {obs }} \ll \sigma / v$, there is a 
random component in the pulse centroid due to the fluctuating pulse width and this can be competitive with the dispersion fluctuation.) We then estimate

$\Delta t \sim 1.9 C_{-4}^{1 / 2} \lambda^{2} D^{1 / 2} v_{7}^{5 / 6} t_{\text {obs }}^{5 / 6} \mu \mathrm{s}$,

where $t_{\mathrm{obs}}$ is measured in years and only the contribution due to dispersion fluctuations has been included. This result agrees with the timing noise previously calculated by Armstrong (1984, see also Rickett 1977) who computes the power spectrum of the relative frequency fluctuations and, equivalently, the Allen variance. Note that, as pointed out by Armstrong, the dispersion measure fluctuations are removable, at least in principle. If this can be accomplished, then there will be a much smaller residual delay $(\sim \Delta \tau)$ caused by geometrical path-length fluctuations. Even this is partly removable by monitoring the flux and pulse width variability. The arrival-time fluctuations considered here are well correlated over a wide frequency range. Equation (5.14) is only an approximate estimate of the timing noise, since we have allowed fot the reduction in the residuals from fitting pulsar parameters like the period, period derivative and position by somewhat arbitrarily cutting off the spectrum at $q_{\min }=2 \pi / v t_{\text {obs }}$. A more careful analysis is being published elsewhere (Blandford, Narayan \& Romani 1984).

Perhaps the most specific prediction of the present model is that the four fluctuating quantities should be cross-correlated as shown in Fig. 4. We can express this in terms of normalized cross-correlation coefficients at zero time lag,

$C_{F \Omega}=\frac{\langle\Delta F \Delta \Omega\rangle}{\Delta F_{\mathrm{rms}} \Delta \Omega_{\mathrm{rms}}}=0.61$

$C_{F \tau}=0.76$

$C_{\Omega \tau}=0.91$.

The flux, angular area and pulse width fluctuations are also quite strongly correlated with the arrival-time fluctuations, but, since $\Delta t_{\text {rms }}$ increases with the duration of the observation as shown by equation (5.14), the normalized correlation coefficient will decrease with time.

We can also use the scattering model to explore the correlation in observing wavelength. Using equations (4.20) and (5.2), we see that the correlation coefficient for flux fluctuations at two different frequencies $\lambda_{1}, \lambda_{2}$ and zero time lag satisfies

$\left\langle\delta F_{1} \delta F_{2}\right\rangle \propto \lambda_{1}^{2} \lambda_{2}^{2}\left(\lambda_{1}^{22 / 5}+\lambda_{2}^{22 / 5}\right)^{-7 / 6}$.

The half-power points are at $\lambda_{2} / \lambda_{1}=(0.5,1.5)$ and we confirm that flux fluctuations should be well correlated in wavelength as appears to be the case (e.g. Helfand et al. 1977). Carrying out a similar calculation for the angular size and pulse width correlations we find that the half-power points of the two correlation functions are located at $\lambda_{2} / \lambda_{1}=(0.7,1.3)$ and $(0.5,1.5)$ respectively. These autocorrelation functions are plotted in Fig. 5. The wavelength correlation of the arrival-time fluctuations again requires a more careful discussioin.

We have thus been able to use our model to compute expressions for the fluctuating quantities assuming a standard Kolmogorov spectrum. Where there is overlap, these expressions are consistent with those given by earlier authors. As discussed by Rickett et al. (1984), the dispersion measure and wavelength dependences (equation 5.9) of the intensity variability time-scale $T_{F}$ is compatible with the obervational data. However, the theory appears to underestimate the strength of the fluctuations. The highly correlated nature of the variation in the intensities of the main pulse, precursor and interpulse of the Crab pulsar over a 3-yr period observed by Rankin et al. (1974) strongly argues in favour of a propagation effect, but the magnitude of the intensity fluctuation (nearly a factor of 10) is incompatible with equation (5.8). Similarly, the observations of Cole et al. (1970) and Helfand et al. (1977) indicate that the flux variability in most pulsars 
extends down to low frequency with an amplitude of typically $\geq 30$ per cent, whereas the calculated variability is somewhat less than this, particularly at long wavelengths. For instance, Helfand $e$ al. measure $\delta F$ to be 0.35 and 0.50 in PSR $0329+54$ and $1919+21$ at $\lambda \sim 2 \mathrm{~m}$, while equation (5.8) with appropriate values of $C_{-4}$ from Cordes et al. (1984) predicts $\delta F$ of only 0.09 and 0.10 respectively The reason for the low predicted variability can be seen from equation (4.24) for the constant $K$. As the wavelength of observation increases, so does the size of the pulsar image $\sigma$, and interstellar lenses of size $\sim \sigma$ which are responsible for the refractive fluctuations become progressively weaker. It thus appears that, if the observed strong (low frequency) variability of pulsars is to be explained as a propagation effect alone, there must be relatively more power in the longer wavelength fluctuations, i.e. the spectral slope $\beta$ must exceed the Kolmogorov value.

\section{2 'CRITICAL' SPECTRUM $(\beta=4)$}

We therefore present expressions for the limiting value for which our calculations can be valid, i.e. $\beta=4$. (The above arguments suggest that $\beta$ may actually exceed 4 , but the calculation techniques developed in this paper are not strictly valid in this regime.) If we again normalize the density fluctuation spectrum to be consistent with Armstrong et al. (1981), then

$Q(q)=Q_{0} q^{-4}, \quad Q_{0}=2 \times 10^{-21} C_{-4} \mathrm{D} \mathrm{cm}^{-7}$

where a scaling factor $C_{-4}$ of order unity is equivalent to $C_{\mathrm{N}}^{2} \sim 10^{-4} \mathrm{~m}^{-6.7}$ as in the Kolmogorov spectrum. We substitute in equation (4.25) to obtain

$K=\frac{1}{\ln \left(430 C_{-4} \lambda^{3} D^{2} / K^{1 / 2}\right)}=\frac{1}{7 \gamma}$

where $\gamma=1$ for $C_{-4}=\lambda=D=1$ and has only a weak logarithmic dependence on $C_{-4}, \lambda$ and $D$. From this we compute as before,

$2 \theta=5 \gamma^{1 / 2} C_{-4}^{1 / 2} \lambda^{2} D^{1 / 2}$ milliarcsec

$\tau_{0}=t_{0}=4 \gamma C_{-4} \lambda^{4} D^{2} \mu \mathrm{s}$

$\Delta v=40 \gamma^{-1} C_{-4}^{-1} \lambda^{-4} D^{-2} \mathrm{kHz}$.

The magnitudes of the long-term fluctuations are

$\delta F=\frac{\Delta F}{F}=0.4 \gamma^{-1 / 2}$

$2 \Delta \theta=0.6 C_{-4}^{1 / 2} \lambda^{2} D^{1 / 2}$ milliarcsec

$\Delta \tau=1.3 \gamma^{1 / 2} C_{-4} \lambda^{4} D^{2} \mu \mathrm{s}$.

The corresponding autocorrelation time-scales are

$T_{F} \sim 0.7 T_{\theta} \sim 0.6 T_{\tau} \sim 25 \gamma^{1 / 2} C_{-4}^{1 / 2} \lambda^{2} D^{3 / 2} v_{7}^{-1}$ day,

the arrival time fluctuation is

$\Delta t \sim 5 C_{-4}^{1 / 2} \lambda^{2} D^{1 / 2} v_{7} t_{\text {obs }} \mu \mathrm{s}$,

the normalized cross-correlation coefficients are

$$
\begin{aligned}
& C_{F \Omega}=0.71 \\
& C_{F \tau}=0.80 \\
& C_{\Omega \tau}=0.94,
\end{aligned}
$$


and, finally, the half-power widths of the two wavelength autocorrelation coefficients for the flux, angular size and pulse-width variations are respectively

$\left\langle\delta F_{1} \delta F_{2}\right\rangle: \lambda_{2} / \lambda_{1}=(0.5,1.9)$

$\left\langle\delta \Omega_{1} \delta \Omega_{2}\right\rangle: \lambda_{2} / \lambda_{1}=(0.7,1.4)$

$\left\langle\delta \tau_{1} \delta \tau_{2}\right\rangle: \lambda_{2} / \lambda_{1}=(0.5,1.8)$.

Thus, a steepening of the slope of the density fluctuation spectrum from $\beta=11 / 3$ to $\beta=4$ increases the predicted flux variation and makes it roughly independent of observing wavelength, in better agreement with observations. There are corresponding changes in the estimates of the other observable quantities as seen by comparing equations (5.20)-(5.31) with (5.3)-(5.17). In fact the actual value of $\beta$ could possibly exceed 4 by a small amount. In this case the scattering is dominated by large-scale density fluctuations and our theory is no longer valid, though the estimates embodied in equations (5.20)-(5.31) are probably still useful and accurate to a factor $\$ 3$. Goodman \& Narayan (1985) present a more careful discussion of intensity fluctuations when $\beta>4$.

\subsection{OBSERVATIONS ON PULSARS}

The angular size variations predicted for a few nearby pulsars with unusually large $C_{-4}$ are probably within the capabilities of low-frequency VLBI. An extreme case, regrettably inaccessible to northern hemisphere VLBI, is the Vela pulsar which lies at a distance of $0.5 \mathrm{kpc}$. The measured pulse broadening at a wavelength of $75 \mathrm{~cm}$ is $\sim 15 \mathrm{~ms}$. The pulsar should therefore subtend an angular diameter of $\sim 300$ milliarcsec at this wavelength. The rms fluctuations in the angular size are expected to be $\sim 30$ milliarcsec for $\beta=4$ and should occur with a decorrelation time $\sim 3 \mathrm{yr}$. (In fact it may be possible to monitor changes in the centroid of the VLBI image which should be of the same order of magnitude as the size changes and should also be correlated with $d F / d T$ and the slope of the drifting patterns in dynamic scintillation spectra, Blandford \& Narayan 1984.)

The predicted fluctuations in pulse widths and arrival times are also measurable in a few pulsars. In particular, in the case of the millisecond pulsar $1937+21$, we can use the measured decorrelation bandwidth of $6.3 \mathrm{kHz}$ at $430 \mathrm{MHz}$ (Cordes \& Stinebring 1984) and the distance estimate $D \sim 5 \mathrm{kpc}$ to estimate that $C_{-4}=0.9$ for $\beta=4$. We then find that the expected pulse-width fluctuation is $\Delta \tau \sim 34 \lambda^{4} \mu \mathrm{s}$ and the rms timing residual is $\Delta t \sim 8 \lambda^{2} t_{\mathrm{obs}} \mu \mathrm{s}$ (taking $v_{7}=0.8$, Cordes, private communication).

Table 1 gives a short list of relatively bright pulsars that appear suitable for monitoring refractive low-frequency variability. For the calculations, $v$ was taken to be the measured pulsar transverse velocity and the wavelengths have been selected so as to give refractive time-scales $\leqslant 1$ yr. PSR 1818-04 seems to be the most interesting for VLBI observations. Several of the pulsars listed are predicted to have significant pulse-width fluctuations at metre and decametre wavelengths. A long-term low-frequency observational programme to look for correlated fluctuations of flux and pulse width in these pulsars appears to be worthwhile. It should be noted that the strengths of fluctuations quoted correspond to $\beta=4$. If the spectrum is still steeper, the fluctuations will increase significantly. On the other hand, it the spectrum is Kolmogorov $(\beta=11 / 3)$, the rms variations will be reduced by a factor $\sim 3$.

Decorrelation bandwidth fluctuations can also be monitored at sub-metre wavelengths, where pulse widths are too small to be measured. An important advantage in going to short wavelengths is that the refractive time-scale goes as $\lambda^{2}$ (or $\lambda^{11 / 5}$ for $\beta=11 / 3$ ) and so most pulsars will have convenient time-scales for observations, leading to a much wider selection of potentially 
Table 1. Examples of pulsars displaying large refractive fluctuations. Values of $C_{-4}$ are from Cordes et al. (1984) and velocities from Lyne $e t$ al. (1982) except those in brackets which have been estimated from scintillation data by Cordes, private communication. Other pulsar parameters are from Manchester $\&$ Taylor (1981). All calculations are for a critical spectrum with $\beta=4$.

\begin{tabular}{|c|c|c|c|c|c|c|c|c|c|c|c|c|}
\hline PSR & $\begin{array}{l}\text { Flux } \\
S_{400}(\mathrm{mJy})\end{array}$ & $D(k p c)$ & $\mathrm{v}\left(\mathrm{km} \mathrm{s}^{-1}\right)$ & $C_{-4}$ & $\lambda(m)$ & $2 \theta$ (mas) & $\tau(\mathrm{ms})$ & $\frac{\sigma}{v}$ (days) & $\frac{\Delta F}{\bar{F}}$ & $2 \Delta \theta$ (mas) & $\Delta \tau(\mathrm{ms})$ & $\frac{\Delta t(m s)}{t_{\text {obs }}(y r)}$ \\
\hline $0329+54$ & 1400 & 2.3 & 229 & 1.1 & 3 & 90 & 3 & 400 & 0.28 & 9 & 0.8 & 0.2 \\
\hline $0531+21$ & 800 & 2.0 & 123 & 2.5 & 1.5 & 30 & 0.3 & 200 & 0.30 & 3 & 0.08 & 0.03 \\
\hline $0833-45$ & 5000 & 0.5 & $(60)$ & 14000 & 0.4 & 90 & 0.6 & 300 & 0.28 & 8 & 0.15 & 0.04 \\
\hline $1133+16$ & 340 & 0.15 & 264 & 11 & 10 & 800 & 20 & 200 & 0.28 & 80 & 4 & 2 \\
\hline $1237+25$ & 160 & 0.33 & 178 & 2.2 & 10 & 600 & 20 & 500 & 0.28 & 50 & 4 & 0.8 \\
\hline $1508+55$ & 125 & 0.73 & 346 & 4.1 & 3 & 100 & 1 & 90 & 0.30 & 10 & 0.3 & 0.3 \\
\hline $1818-04$ & 170 & 1.5 & 194 & 89 & 1 & 70 & 1 & 300 & 0.28 & 7 & 0.3 & 0.1 \\
\hline $1937+21$ & 100 & 5.0 & (80) & 0.9 & 0.7 & 6 & 0.03 & 200 & 0.33 & 0.7 & 0.007 & 0.004 \\
\hline
\end{tabular}


interesting pulsars. The decorrelation bandwidth is expected to be anticorrelated with the pulse width because of the approximate relation $2 \pi \tau \Delta v \sim 1$, and so the present results on $\Delta \tau$ could be borrowed to make rough estimates of the magnitude of the fluctuation in $\Delta v$. However, the present geometrical optics approach may need to be modified to handle diffraction phenomena. We plan to discuss this in a future paper.

\section{Extragalactic radio sources}

The majority of low-frequency variable extragalactic radio sources are at high Galactic latitude and model (b) of Appendix A is appropriate. If we assume that the scale height of the electron density layer is $\sim 0.5 \mathrm{kpc}$ (Cordes et al. 1984) and that the fluctuations are spatially uniform, then the time-scale for the variability should be $\sim 30 \lambda^{2} \operatorname{cosec}^{3 / 2}(b) v_{7}^{-1}$ day (assuming $\beta=4$ and $C_{-4}=1$ ), where now $v_{7}$ is an effective velocity including the motion of the Earth and the medium. Variability should only occur for $\lambda \geq 10 \mathrm{~cm}$. These predictions are compatible with the observed range of variability wavelengths and time-scales, as concluded by Rickett et al. (1977). The correct choice of the relative velocity is problematical, however, and if we add to this uncertainty the real possibility that the fluctuations may be spatially inhomogeneous, then there is probably enough scatter to mask the rather weak predicted dependence of the variability time-scale on Galactic latitude. The scaling with wavelength may also be confused because we know that there must be intrinsic variability at centimetre wavelengths and that this must contribute to the observed variation at intermediate wavelengths. The predicted interstellar scattering angular sizes $\left[2 \theta \sim 4 \lambda^{2} \operatorname{cosec}^{1 / 2}(b)\right.$ milliarcsec] are probably just too small to influence the source structures derived from normal ground-based VLBI (although they should be taken into account in the design of the proposed $Q U A S A T$ mission). Low-frequency VLBI should be able to monitor the fluctuations in the position and angular size of the sources. It would be interesting to look for correlations of these with flux variations.

\section{Conclusions}

It has been suggested that the $\sim$ monthly variability observed in pulsars and compact extragalactic radio sources could be largely an extrinsic, interstellar effect. This is an appealing idea for which there is already some observational support. In this paper, we point out that it should be testable using careful observations of selected pulsars.

The long time-variability is attributed to long-wavelength electron density fluctuations. We have introduced a simple model that localizes all these fluctuations in a thin screen and computes their focusing and defocusing effects on a beam of radio waves that is already broadened by smaller scale fluctuations. This geometric approach is only valid if the small-scale scattering exceeds the large-scale scattering, a condition that is at best marginally satisfied in the interstellar medium.

When the beam is focused, the flux, pulse width and angular size should simultaneously increase and the pulse should arrive early. We have estimated the associated auto- and cross-correlation coefficients for fluctuations in these quantities together with their variations with time and wavelength difference. We have also suggested some candidate pulsars (Table 1) in which to seek these correlations. If the correlations are found then they should confirm the importance of interstellar refraction. They should also motivate more detailed calculations of these effects.

Since strong flux variation is observed at low frequency, we have tentatively concluded that the amplitude of the spectrum of the fluctuations should have a spectral slope somewhat steeper than the traditional 'Kolmogorov' value, $\beta=11 / 3$. It may exceed the 'critical' value of 4 , in which case 
the calculations of this paper are strictly invalid. Roberts \& Ables (1982) suggested that $\beta$ may be greater than 4 because of the occurrence of periodicities and multiple drift slopes in dynamic scintillation spectra, arguing that these arose from the interference of a few beams (see also Ewing et al. 1970). An immediate consequence of this picture is that the intensity variation across the pulsar image will not be the smooth Gaussian that we have assumed but may be 'patchy' with a few dominant bright spots. VLBI maps of pulsar images should clarify this issue.

There are two further implications of the ideas presented in this paper that are of immediate observational relevance. First, arrival-time fluctuations may limit the accuracy with which the millisecond pulsar $1937+21$ can be timed. This will in turn limit its usefulness as a sensitive detector of long-wavelength gravitational radiation (e.g. Romani \& Taylor 1983; Armstrong 1984; Blandford et al. 1984). We believe that it may be possible to correct for some of these effects by carefully monitoring the flux, pulse width and dispersion measure variation. We plan to discuss this elsewhere. The second issue concerns the direct influence of the refractive effects on the diftractive scintillation. In particular, variations in the decorrelation bandwidth and the rate of frequency drifting of the scintillation pattern can be used as a more sensitive probe of interstellar refraction. As the analysis of these effects goes beyond the purely geometrical optics discussion of the present paper, we have deferred this also to a subsequent publication.

In conclusion, we urge pulsar observers to schedule their monitoring programs so that the predicted cross-correlations can be detected or shown to be absent. Whatever the outcome of such investigations, they should tell us more about the interstellar medium and the nature of extragalactic radio sources.

\section{Acknowledgments}

We are indebted to many colleagues, notably J. Armstrong, J. Cordes, J. Goodman, A. Readhead, R. Romani and J. Taylor, for invaluable discussions. We thank B. J. Rickett for detailed comments on the manuscript. Support by the National Science Foundation under grant AST 82-13001 and the Alfred P. Sloan Foundation is gratefully acknowledged.

\section{References}

Abramowitz, M. \& Stegun, J. A., 1970. Handbook of Mathematical Functions, Dover Publications, New York. Alcock, C. R. \& Hatchett, S. P., 1978. Astrophys. J., 222, 456.

Armstrong, J. W., 1984. Nature, 307, 527.

Armstrong, J. W., Cordes, J. M. \& Rickett, B. J., 1981. Nature, 291, 561.

Blandford, R. \& Narayan, R., 1984. Proceedings of the Workshop on Millisecond Pulsars, p. 310, eds Reynolds, S. P. \& Stinebring, D. R., Green Bank.

Blandford, R., Narayan, R. \& Romani, R. W., 1984. J. Astrophys. Astr., 5, 369.

Cole, T. W., Hesse, H. K. \& Page, C. G., 1970. Nature, 225, 712.

Cordes, J. M. \& Stinebring, D. R., 1984. Astrophys. J., 277, L53.

Cordes, J. M., Weisberg, J. \& Boriakoff, V., 1984. Preprint.

Ewing, M. S., Batchelor, R. A., Friefeld, R. D., Price, R. M. \& Staelin, D. J., 1970. Astrophys. J., 162, L169.

Gapper, G. R. \& Hewish, A., 1981. Mon. Not. R. astr. Soc., 197, 209.

Goodman, J. \& Narayan, R., 1985. Mon. Not. R. astr. Soc., in press.

Helfand, D. J., Fowler, L. A. \& Kuhlman, J. V., 1977. Astr. J., 82, 701.

Hewish, A., 1980. Mon. Not. R. astr. Soc., 192, 799.

Lyne, A. G., Anderson, B. \& Salter, M. J., 1982. Mon. Not. R. astr. Soc., 201, 503.

Manchester, R. N. \& Taylor, J. H., 1977. Pulsars, Freeman, San Francisco.

Manchester, R. N. \& Taylor, J. H., 1981. Astrophys. J., 86, 1953.

Rankin, J. M., Payne, R. R. \& Campell, D. B., 1974. Astrophys. J., 193, L71.

Rickett, B. J., 1977. Ann. Rev. Astr. Astrophys., 15, 479.

Rickett, B. J., Coles, W. A. \& Bourgois, G., 1984. Astr. Astrophys., 134, 390.

Roberts, J. A. \& Ables, J. G., 1982. Mon. Not. R. astr. Soc., 201, 1119.

Romani, R. \& Taylor, J. H., 1983. Astrophys. J., 265, L35. 
Scheuer, P. A. G., 1968. Nature, 218, 920.

Shishov, V. I., 1974. Soviet Astr., 17, 598.

Sieber, W., 1982. Astr. Astrophys., 113, 311.

Woolf, N. J., 1982. Ann. Rev. Astr. Astrophys., 20, 367.

\section{Appendix A}

We study the scattering of rays by the inhomogeneities in the interstellar medium in the limit when different rays within the image suffer uncorrelated deflections. Let the rays be scattered through a small angle $\Delta \xi$ on traversing a distance $\Delta z$ of the medium. If the scattering is isotropic about the ray direction, then it can be described by a mean scattering rate, $\psi(z)=\left\langle(\Delta \xi)^{2} / \Delta z\right\rangle$. Define $\theta$ to be the angle between the local direction of propagation of a ray and the radius vector from the source. Then $\theta^{2}$ can be shown to satisfy the following differential equation

$\frac{1}{z^{2}} \frac{d\left(z^{2} \theta^{2}\right)}{d z}=\psi(z)$

Hence, integrating along the line-of-sight from the observer to the pulsar at distance $D$,

$\theta^{2}=\frac{1}{D^{2}} \int_{0}^{D} d z z^{2} \psi(z)$

A point source will have a circular Gaussian brightness distribution of angular radius $\theta$. Likewise, the mean geometric time delay satisfies

$c \frac{d t}{d z}=\frac{\theta^{2}}{2}$.

Solving, we obtain ( $c f$. Alcock \& Hatchett 1978)

$t=\frac{1}{2 c D} \int_{0}^{D} d z z(D-z) \psi(z)$

We locate the equivalent screen at distance

$L=2 c t / \theta^{2}$

and adjust the strength of the inhomogeneities on the screen so that the mean angular size of the image becomes $\theta$.

For illustration purposes, we shall consider two simple scattering models.

(a) The scattering is uniform between Earth and a pulsar lying in the Galactic plane. In this case,

$L=D / 2$

and the strength of the fluctuations on the screen is equivalent to collapsing a slab of the extended medium of thickness $L^{\prime}$, where

$L^{\prime}=D / 3$.

(b) The scattering is distributed exponentially with scale height $H \operatorname{cosec}(b)$, and the pulsar is located at a height much greater than $H$ above the Galactic plane. In this case

$L=L^{\prime}=H \operatorname{cosec}(b)$. 


\section{Appendix B}

We present here the correlation coefficients for the normalized fluctuations calculated using the method described in Section 4. If we adopt a power-law spectrum of density fluctuations, $Q=Q_{0} q^{-\beta}$, then we find that

$\langle\delta F(\mathbf{x}) \delta F(\mathbf{x}+\mathbf{s})\rangle=K h_{1}$

$\langle\delta F(\mathbf{x}) \delta \Omega(\mathbf{x}+\mathbf{s})\rangle=K\left(h_{1}-1 / 4 h_{2}\right)$

$\langle\delta F(\mathbf{x}) \delta t(\mathbf{x}+\mathbf{s})\rangle=-2 K\left(h_{0}-1 / 2 h_{1}+1 / 8 h_{2}\right)$

$\langle\delta F(\mathbf{x}) \delta \tau(\mathbf{x}+\mathbf{s})\rangle=3 / 2 K\left(h_{1}-1 / 3 h_{2}+1 / 48 h_{3}\right)$

$\langle\delta \Omega(\mathbf{x}) \delta \Omega(\mathbf{x}+\mathbf{s})\rangle=K\left(h_{1}-1 / 2 h_{2}+1 / 16 h_{3}\right)$

$\langle\delta \Omega(\mathbf{x}) \delta t(\mathbf{x}+\mathbf{s})\rangle=-2 K\left(h_{0}-3 / 4 h_{1}+1 / 4 h_{2}-1 / 32 h_{3}\right)$

$\langle\delta \Omega(\mathbf{x}) \delta \tau(\mathbf{x}+\mathbf{s})\rangle=3 / 2 K\left(h_{1}-7 / 12 h_{2}+5 / 48 h_{3}-1 / 192 h_{4}\right)$

$\langle\delta t(\mathbf{x}) \delta t(\mathbf{x}+\mathbf{s})\rangle=K\left(h_{-1}-h_{0}+1 / 2 h_{1}+1 / 8 h_{2}+1 / 64 h_{3}\right)$

$\langle\delta t(\mathbf{x}) \delta \tau(\mathbf{x}+\mathbf{s})\rangle=3 K\left(h_{0}-5 / 6 h_{1}+15 / 48 h_{2}-5 / 96 h_{3}+1 / 384 h_{4}\right)$

$\langle\delta \tau(\mathbf{x}) \delta \tau(\mathbf{x}+\mathbf{s})\rangle=9 / 4 K\left(h_{1}-2 / 3 h_{2}+11 / 72 h_{3}-1 / 72 h_{4}+1 / 2304 h_{5}\right)$,

where $K$ and $h_{n}(s)$ are defined by equations (4.17), (4.18). The functions $h_{n}(s)$ only exist for $n>\beta-4$. Consequently the expression for $\langle\delta t(\mathbf{x}) \delta t(\mathbf{x}+\mathbf{s})\rangle$ is only valid for $\beta<3$ and the cross-correlations $\langle\delta t(\mathbf{x}) \delta A(\mathbf{x}+\mathbf{s})\rangle$ for $\beta<4$. When these conditions are violated, then we must cut off the integral in equation (4.8) at $q=q_{\min } \sim 2 \pi / v t_{\text {obs }}$ as described in Section 5.

We can also derive expressions for the auto-correlations of $\delta F, \delta \Omega, \delta \tau$ with respect to changes in the observing wavelength. Evaluating these at $s=0$, we obtain

$$
\begin{aligned}
& \left\langle\delta F\left(\lambda_{1}\right) \delta F\left(\delta_{2}\right) \propto \frac{\lambda_{1}^{2} \lambda_{2}^{2}}{\left[\sigma_{1}^{2}+\sigma_{2}^{2}\right]^{(6-\beta) / 2}}\right. \\
& \left\langle\delta \Omega\left(\lambda_{1}\right) \delta \Omega\left(\lambda_{2}\right)\right\rangle \propto \frac{\lambda_{1}^{2} \lambda_{2}^{2}\left[2(\beta-4)\left(\sigma_{1}^{2}+\sigma_{2}^{2}\right)^{2}+(8-\beta)(6-\beta) \sigma_{1}^{2} \sigma_{2}^{2}\right]}{4\left(\sigma_{1}^{2}+\sigma_{2}^{2}\right)^{(10-\beta) / 2}} \\
& \left\langle\delta \tau\left(\lambda_{1}\right) \delta \tau\left(\lambda_{2}\right)\right\rangle \propto \frac{\lambda_{1}^{2} \lambda_{2}^{2}}{\left(\sigma_{1}^{2}+\sigma_{2}^{2}\right)^{(6-\beta) / 2}}\left\{\frac{2 \beta-9}{3}+\frac{(6-\beta)(8-\beta)}{6\left(\sigma_{1}^{2}+\sigma_{2}^{2}\right)^{2}}\left[\frac{\left(\sigma_{1}^{4}+\sigma_{2}^{4}\right)}{2}+\frac{(\beta-2)}{3} \sigma_{1}^{2} \sigma_{2}^{2}\right.\right. \\
& \left.\left.\quad+\frac{1}{24} \frac{\sigma_{1}^{4} \sigma_{2}^{4}}{\left(\sigma_{1}^{2}+\sigma_{2}^{2}\right)^{2}}(10-\beta)(12-\beta)\right]\right\}
\end{aligned}
$$

where $\sigma \propto \lambda^{\beta /(\beta-2)}$ and $\sigma_{1,2}=\sigma\left(\lambda_{1,2}\right)$. These autocorrelations can be normalized using equations (B1), (B5) and (B10). 